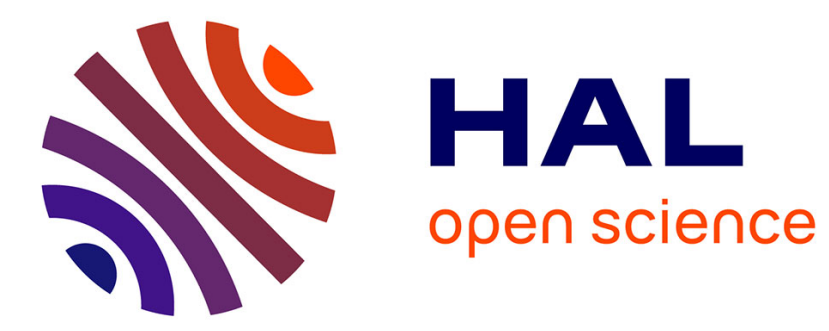

\title{
Different scenarios of shrinking surface soap bubbles
}

\author{
Mattéo Clerget, Alexandre Delvert, Laurent Courbin, Pascal Panizza
}

\section{To cite this version:}

Mattéo Clerget, Alexandre Delvert, Laurent Courbin, Pascal Panizza. Different scenarios of shrinking surface soap bubbles. American Journal of Physics, 2021, 89 (3), pp.244-252. 10.1119/10.0002348 . hal-03190298

\section{HAL Id: hal-03190298 \\ https://hal.science/hal-03190298}

Submitted on 29 Nov 2021

HAL is a multi-disciplinary open access archive for the deposit and dissemination of scientific research documents, whether they are published or not. The documents may come from teaching and research institutions in France or abroad, or from public or private research centers.
L'archive ouverte pluridisciplinaire $\mathbf{H A L}$, est destinée au dépôt et à la diffusion de documents scientifiques de niveau recherche, publiés ou non, émanant des établissements d'enseignement et de recherche français ou étrangers, des laboratoires publics ou privés. 


\title{
Different scenarios of shrinking surface soap bubbles
}

\begin{abstract}
Mattéo Clerget, ${ }^{*}$ Alexandre Delvert, ${ }^{*}$ Laurent Courbin, ${ }^{\dagger}$ and Pascal Panizza ${ }^{\ddagger}$ Univ Rennes, CNRS, IPR (Institut de Physique de Rennes) - UMR 6251, F-35000 Rennes, France (Received; Accepted)

We discuss a simple experiment investigating the shrinkage of surface soap bubbles sitting on a thin solid plate with a circular orifice located under the apex of the bubble. We identify three different shrinking regimes, the occurrence of which depends on a combination of key parameters that include the ratio between initial bubble and orifice sizes and physicochemical properties of the fluid system. For low-viscosity liquids and/or large ratios, a bubble remains quasi-hemispherical as shrinking proceeds. By contrast, for liquids with sufficiently large viscosities and/or small geometric ratios, a bubble seeks the shape of a spherical cap while the air inside it escapes through the orifice. In this case, shrinking proceeds with a bubble foot that either recedes over time or does not move for the largest viscosities and/or smallest ratios. We use basic physical arguments to rationalize the three identified regimes and to explain the shrinking dynamics. Specifically, this model which captures observations and measurements is based on Bernoulli's principle for the air flow, volume conservation and a friction law that accounts for viscous dissipation at the moving bubble foot.
\end{abstract}

\section{INTRODUCTION}

The draining of a tank initially filled with a liquid is a common problem that impacts processes in many fields that include food and oil industries. It has been investigated starting from the pioneering work by Torricelli who established a well-known inviscid law for the fluid velocity ${ }^{1}$ until a recent work that has revealed the importance of surface wettability on the speed of drainage. ${ }^{2}$ Also, students are familiar with this problem which is useful in the classroom to introduce both the notion of free fall and the steady-state Bernoulli's principle. Here, we investigate a variant of the Torricelli's problem, that is, the case of air escaping a deformable tank by working with a soap bubble sitting on a solid substrate that has a circular orifice located under the apex of the bubble. Also it is worthwhile noting that the experiment described in this article can be seen as a variant of a deflating bubble experiment ${ }^{3}$ in which the bubble foot would be allowed to move. In a deflating bubble experiment, a hemispherical bubble formed at the end of a tube deflates with a pinned foot when the tube's other end is opened. Since a long and narrow tube is used in this study, another difference between experiments resides in the air flow that satisfies Poiseuille's law ${ }^{3}$ rather than the Bernoulli's principle.

Bubbles are important for a variety of reasons. For instance, centimeter-sized floating bubbles made by blowing air onto soap films are useful for entertainment and scientific purposes and they are excellent tools in the classroom that can aid the learning of physics. For these reasons, they have been studied for centuries ${ }^{4-6}$ although their formation mechanism has been explained only recently. ${ }^{7,8}$ Also, at smaller scales, bubbles with sizes in the $\mu \mathrm{m}-\mathrm{mm}$ range are employed in diverse applications in fields that range from material science to medicine and biology. ${ }^{9}$ In Nature, surface bubbles with sizes in the $\mathrm{mm}$-cm range and sitting on a liquid-gas interface play a key role in processes occurring at the surface of oceans. These bubbles are indeed important for climate as their rupture can produce aerosol droplets that contribute to the formation of sea spray. ${ }^{10-12}$

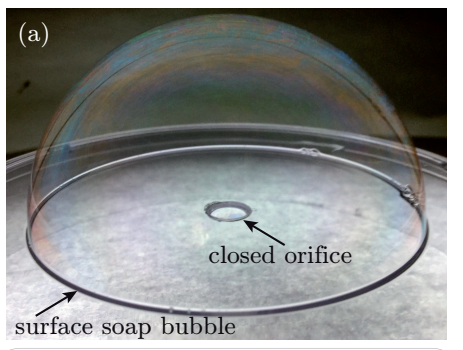

(b)

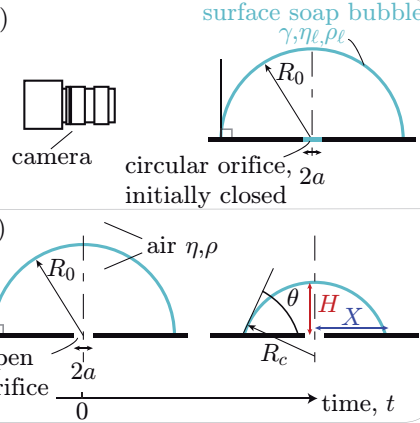

FIG. 1. (a) Photograph illustrating our experiment in which a surface soap bubble sits on a thin plate above an orifice that is initially closed; the $6 \mathrm{~mm}$ orifice diameter indicates the scale. Schematic of our (b) setup and (c) experiment defining the variables at play. (b) A bubble is blown on the surface over an orifice of radius $a$ until it reaches a desired value of its radius $R_{0}$. This process forms a soap film that seals the orifice which is opened (c) at the origin of time $t$ of an experiment and we record the temporal variations of the bubble's radius of curvature $R_{c}$, its height $H$ at its center and the distance $X$ describing the position of the bubble's foot.

Not surprisingly, the rupture of such surface bubbles 

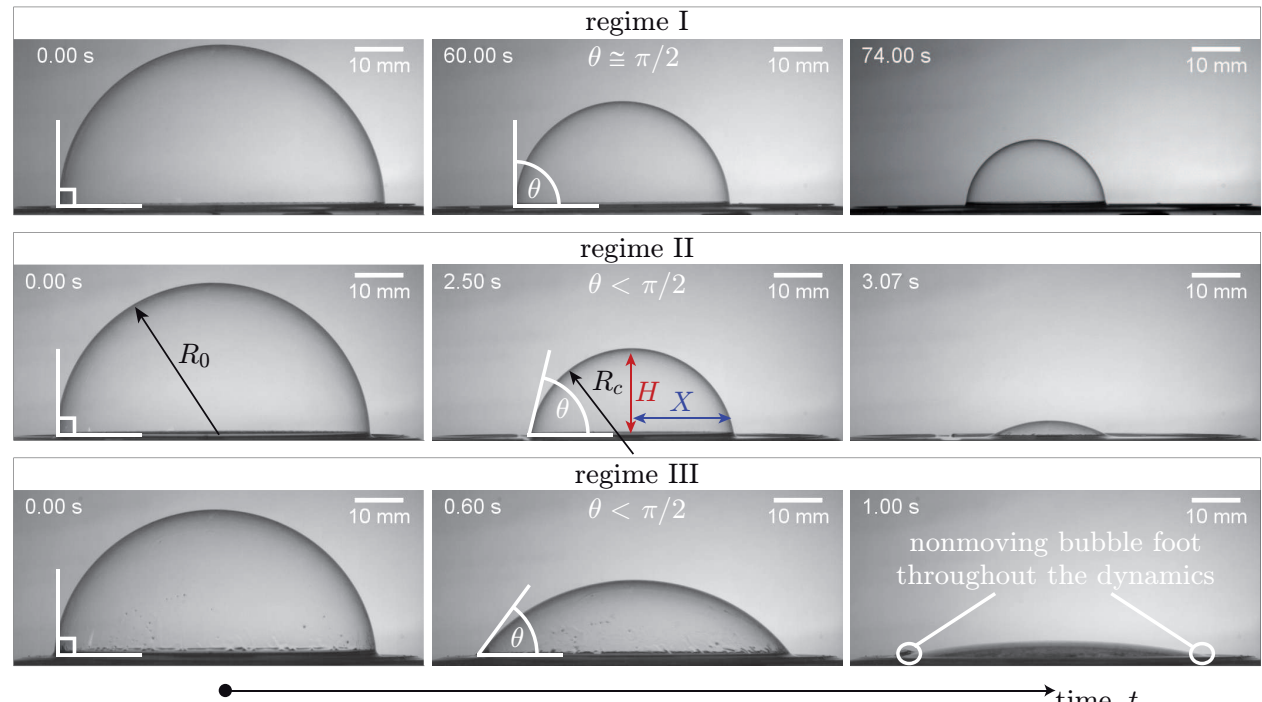

$\rightarrow$ time, $t$

FIG. 2. Sequence of images illustrating from top to bottom the three regimes seen experimentally; these images are taken from the three movies provided as Supplementary Material. ${ }^{22}$ Top row: A bubble remains hemispherical as shrinking occurs in regime I. Middle row: A bubble seeks the shape of a spherical cap when shrinking occurs in regime II. Bottom row: In regime III, a bubble collapses on itself with a distance $X$ that remains unchanged throughout the dynamics. For these experiments, the initial radius of the bubble is fixed and equal to $R_{0}=32 \pm 1 \mathrm{~mm}$. The values of the radius $a$ of the orifice and liquid viscosity $\eta_{\ell}$ are respectively : (top) $0.5 \mathrm{~mm}$ and $1 \mathrm{mPa} \mathrm{s}$, (middle) $2 \mathrm{~mm}$ and $1 \mathrm{mPa}$ s and (bottom) $5 \mathrm{~mm}$ and $22 \mathrm{mPa}$.

and subsequent phenomena have been widely studied by the physics community for the past decade. ${ }^{13-15}$ Also, centimeter-sized surface bubbles can serve as model systems for other types of flows seen in Nature. Indeed, the flow properties of isolated vortices that can appear on the surface of such bubbles bear analogies with those of hurricanes ${ }^{17,18}$ Here, we work with these surface bubbles which are not only important to understand natural processes but also easier to manipulate than those seen at smaller scales. For instance, we have recently elucidated the formation mechanism of centimeter-sized bubbles produced by plunging thin films of liquid in a pool. ${ }^{19}$ In fact, the experiments described in this article do not require sophisticated equipment and they could be easily performed at low cost by students as a laboratory activity. Students would need a solution for blowing bubbles, a straw, a smartphone camera to record the dynamics, Petri dishes that could be used as thin plates and a dril with drill bits of different sizes. In addition, this experiment will give students an opportunity to learn about an intrinsic property of fluid-fluid interfaces, that is, surface tension. This fluid parameter is the interfacial energy required to increase the surface of an interface by one unit At the curved liquid-air interfaces forming a bubble, surface tension can also be seen as a force per unit length which is at the origin of a pressure difference between the inside and the outside of a bubble. This overpressure existing inside drops and bubbles is notoriously known as the Laplace pressure. In our study, because of these properties, a bubble empties itself into the atmosphere as the air inside it flows through the open orifice from the high pressure (inside the bubble) to the low pressure (outside the bubble).

In our experiments, three distinct shrinking regimes can be observed depending on the geometric and physicochemical variables at play (see Fig. 1 and Fig. 2 for a description of our experiment and illustrative examples of the different shrinking regimes). In the first two regimes, an initially hemispherical bubble either remains quasi-hemispherical or seeks the shape of a spherical cap as shrinkage proceeds with a bubble foot that recedes throughout the dynamics. In the third regime, bubbles flatten over time with a nonmoving bubble foot. Combining Bernoulli's principle for the air flow, conservation of the air flow rate and friction acting on the bubble's foot, our modeling work concurs well with experiments.

\section{EXPERIMENTS}

At first glance, these simple experiments seem appropriate for introductory physics laboratories. However, they require knowledge of fluid dynamics, dimensional analysis and differential equations. Thus they should 
be more appropriate for upper-division students. The regime in which a bubble remains quasi-hemispherical during an experiment could be a good project for undergraduate physics students. Indeed, we show in this case that the shrinking dynamics is described by a firstorder ordinary differential equation that can be solved analytically by making an assumption easily met experimentally. We find an excellent agreement between experimental data and predictions of the temporal variations of the bubble size and time at which shrinking ends. This regime is easily observed with a large bubble $(\gtrsim 30 \mathrm{~mm}$ in diameter) shrinking on a plate having a small hole $(\lesssim 1 \mathrm{~mm}$ in diameter) with a soap solution consisting of a few drops of dishwashing detergent diluted in tap water. The slow dynamics observed in this regime could be recorded with a smartphone video camera. The time at which shrinking ends would then be obtained by a direct observation of a movie and the temporal variations of a bubble's size would be measured using a free image processing program such as Tracker ${ }^{20}$ or ImageJ. ${ }^{21}$

The experiments and analysis described in the second part of the article (shrinking spherical caps) might be more appropriate for graduate students. Indeed, in this case, a force accounting for the friction acting on a bubble's foot during shrinkage is introduced in the modeling work so that calculations get slightly more involved. The resulting ODE describing an experiment is then solved numerically. This would be a good computational exercise for graduate physics students. Also, in the case of shrinking spherical cap, the variations of three different primary outcomes (rather than one) can be measured, which makes a good image analysis program needed.

To summarize, the main purpose of our article is to report experimental findings and modeling work that could be used as a laboratory activity for both undergraduate or graduate physics major courses. The exact content of the activity would depend on the level of experience of the students. The physics teacher would have a choice between several activities discussed throughout this paper and summarized in the four-item list below. These items are sorted in ascending order of required level:

Observations: Least experienced students could try to identify the three different regimes that can be observed with the naked eye. Using different liquids and surfaces with holes of various sizes they could be encouraged to guess which physicochemical properties of a liquid and geometric parameters of the problem control the occurrence of a regime.

Building an experimental setup: After gathering the equipment needed to conduct experiments, i.e. a solution for blowing bubbles, a camera, a light source, thin plates with holes and a needle to puncture a film sealing an orifice, students could build an experimental setup that would allow them to record movies which can be analyzed using a free image processing software. They should pay particular attention to the position of the camera and lighting conditions and they should make sure the plates are leveled. Students would then be able to visualize on a computer screen the regimes seen experimentally with the naked eye. Focusing on regime I, they could eventually try to analyze a movie with the aforementioned software to obtain the temporal variations of the geometric quantities characterizing a bubble.

Regime I, interpretation of the results and the importance of approximations in physics: Based on their guess of the fluid and geometric key parameters at play (first item on this list), students should be given a chance, and time, to try to explain the physics behind their measurements in regime I. The physics teacher could guide students as they construct their understanding and he/she could introduce the model of the experiment after some time if needed. The students could then be asked to try to find an approximation (as explained in our article) that would make the determined first-order differential equation solvable analytically. Ultimately, they would then conduct several experiments within this limit and try to adjust the results with the analytical solution of the simpler form taken by the ODE.

Regime II and III, custom-written image processing and numerical simulations: More experienced students could pursue the project with the experimental and theoretical investigations of regimes II and III. The script of our image processing program could help as a handout for the image processing. ${ }^{22}$ The teacher would likely provide the students with the expression of the viscous force and students would then establish the differential equations driving the dynamics themselves and they could solve them numerically and compare the resulting predictions to their experimental results.

Figure 1 shows the experimental setup and defines the variables at play in our experiment. To prepare a surface bubble (Figure 1(a) shows a photograph of such a bubble), we first place a soap film at the end of a circular nozzle of diameter $1 \mathrm{~cm}$ by dipping the nozzle into one of the soap solutions described below. The soap film is then curved by injecting air at controlled pressure through the nozzle with a flow control system (Fluigent). The contact between the curved film and the plate creates a surface bubble which spreads over the substrate that is initially wetted with the same solution until a desired bubble radius $R_{0}=10-35 \mathrm{~mm}$ is reached. As a drilled plate, we use the bottom surface of a $100 \times 20 \mathrm{~mm}$ Petri dish (Falcon). Wetting the solid substrate with the soap solution produces a thin film of liquid having a homogeneous thickness in the range $15-25 \mu \mathrm{m}$ and a size that is made larger than the prepared bubble; film thicknesses are measured with a spectrometer (Avantes AvaSpec-2048) in our study. The prepared bubble which rests on a liquid film of the soap solution is hemispherical as it is at equilibrium with its surroundings, i.e., the soap film at the level of the bubble's foot is perpendicular to the horizontal wetted substrate. ${ }^{23,24} \mathrm{~A}$ bubble is made of a soap solution consisting of $2 \mathrm{wt} . \%$ of dishwashing liquid (Palmolive Original, Colgate-Palmolive) in $98 \mathrm{wt} \%$ Milli-Q water to which an amount (either 0 , or 40 , or 70 wt.\%) of glycerol (Sigma-Aldrich) is added. The liq- 
uid dynamic viscosity and density are $\eta_{\ell}$ and $\rho_{\ell} . \gamma$ is the air-liquid surface tension. Table I summarizes the values of $\eta_{\ell}$ and $\gamma$ for the three soap solutions containing different amount of glycerol. The air density and dynamic viscosity are $\rho$ and $\eta$, respectively. The apex of the bubble is located over the center of the circular orifice of radius $a=0.5-6.5 \mathrm{~mm}$ that is initially closed. Indeed, during the formation of a surface bubble, its spreading over the plate leaves a soap film on the orifice which seals it. In our experiments, an orifice is drilled in a thin plate whose thickness is negligible compared to the radius $a$. For fixed bubble and hole sizes and a given liquid, we find that experiments conducted with different bubble and film thicknesses give the same shrinking dynamics; the thickness of the film forming a bubble is in the range $2-10 \mu \mathrm{m}$ in our experiments. This point should further facilitate implementing the experiment as a project for students. In addition, we have verified that results obtained with bubbles blown by mouth were identical to those provided by our setup.

TABLE I. Dynamic viscosities and air-liquid surface tensions of the three soap solutions prepared with different amounts of glycerol as indicated. The quantities were measured at $20^{\circ} \mathrm{C}$.

\begin{tabular}{lll}
\hline \hline glycerol concentration (wt.\%) & $\eta_{\ell}(\mathrm{m} \mathrm{Pas})$ & $\gamma(\mathrm{m} \mathrm{N} / \mathrm{m})$ \\
\hline 0 & 1 & 25 \\
40 & 4 & 25 \\
70 & 22 & 30 \\
\hline \hline
\end{tabular}

A bubble begins to collapse, i.e., the air begins to escaped out of the bubble, when the orifice is opened (i.e. rapidly punctured with a needle) at the origin of time $t$ and we record with a camera (Photron Fastcam SA3) the temporal evolution of the three main outcomes of the experiment: The maximal height $H$ of a bubble measured between its apex and the center of the orifice, the bubble's radius of curvature $R_{c}$ and the distance $X$ describing the position of a bubble's foot (see Fig. 1 and Fig. 2 defining variables at play). Images of an experiment are analyzed using a custom-written MATLAB software; an annotated script of this software is provided as Supplementary Material. ${ }^{22}$ In our study, the contact angle that the surface of the bubble makes with the substrate at the level of the bubble's foot is $\theta$. As illustrated in Fig. 2 for experiments conducted for a fixed initial bubble radius $R_{0}=32 \pm 1 \mathrm{~mm}$, two of the three soap solutions and three holes of different radii, three different shrinkage scenarios described below can be observed.

For an experiment conducted with the liquid having the lowest viscosity and the smallest orifice of the studied set, i.e. the largest ratio between initial bubble and orifice sizes, a bubble remains quasi-hemispherical as shrinking proceeds. This is regime I shown in the top row of images in Fig. 2, an experiment for which $\theta=89 \pm 1^{\circ}$ throughout the dynamics. In our study, we refer to the quasi-hemispherical bubbles seen in regime I as hemispherical bubbles for the sake of simplicity. As shown in the middle sequence of photographs in Fig. 2, a bubble

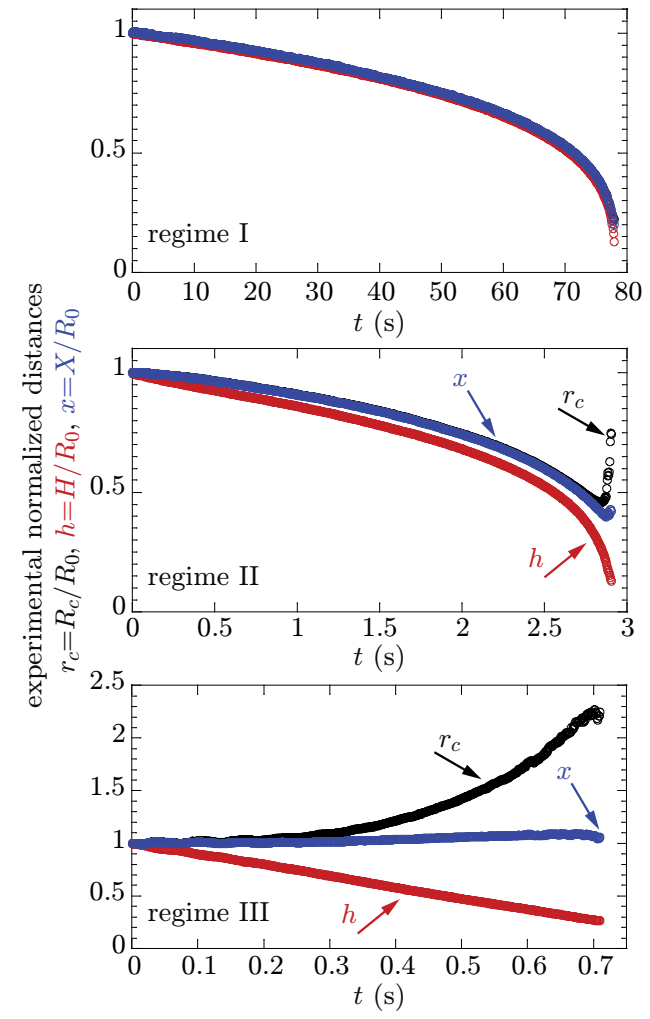

FIG. 3. Experimental variations with time of the normalized distances describing a bubble: The radius of curvature $r_{c}=R / R_{0}$, height $h=H / R_{0}$ and distance $x=X / R_{0}$ that correspond to the three regimes shown in the sequences of images in Fig. 2 and the three movies provided as Supplementary Material. ${ }^{22}$ Parameters are identical to those of Fig. 2.

made of the same solution seeks the shape of a spherical cap as shrinking proceeds when the size of the orifice is increased. This regime is herein denoted regime II. In a third regime (regime III) seen for the liquid having the largest viscosity and the orifice with the largest value of $a$ in Fig. 2, as the air escapes through the orifice, the dynamics is characterized by a bubble collapsing on itself with a nonmoving bubble's foot (see bottom row of images in Fig. 2). For the whole study, we nondimensionalize the problem with $R_{c}=R_{0} r_{c}, H=R_{0} h$ $X=R_{0} x=$ and $t=\tau \varepsilon$ where $\tau$ is a characteristic time of the experiment that is defined in section III A. Figure 3 shows temporal evolutions of the normalized distances that correspond to the sequences of images reported in Fig. 2. For this investigation, image processing captures most of the shrinking dynamics in any of the three regimes (see Fig. 3) but it fails to describe the last 
moments of an experiment. As a result, the experimental time $t_{\text {end }}$ at which shrinking ends is obtained by a direct observation of the movie of an experiment.

We rationalize these experimental observations and measurements in section III and we compare the resulting predictions to systematic experiments in section IV.

\section{MODEL OF THE FLOW}

In what follows, we model the different shrinking dynamics obtained in the three regimes seen experimentally (Figs. 2 and 3). Firstly, in section III A, we begin by the regime I in which the bubbles remain hemispherical while it shrinks. Secondly, we rationalize in section III B the dynamics seen in regime II, that is, the regime in which the shape of a bubble is a spherical cap as shrinking proceeds. Lastly, we rationalize in section III C our findings in regime III where a bubble's foot is pinned during an experiment. For each of the three regimes seen experimentally, we model the dynamics assuming the air flow satisfies Bernoulli's principle. Indeed, for the investigated flow configuration, it seems reasonable to consider that air is inviscid so that dissipative effects by viscous forces can be neglected and air flow can be described with the Bernoulli's equation as shown below. By contrast, viscous dissipation impacts the motion of a bubble's foot in our experiment and a Reynolds number associated with this flow can be defined. This Reynolds number $\mathcal{R}_{\ell}$ can be written $e \rho_{\ell} \dot{X} / \eta_{\ell}$ where $\rho_{\ell}$ and $e$ are the liquid density and thickness of the liquid film under a bubble, respectively. For the plot illustrating Regime II in the middle panel of Fig. 2, we find that $\mathcal{R}_{\ell}$ is $O\left(10^{-1}\right)$ throughout the dynamics which indicates that viscous effects are indeed important at the bubble's foot; we have used an average film thickness $e=20 \mu \mathrm{m}$ to determine this estimate.

\section{A. Regime I: the case of hemispherical bubbles $(\theta \cong \pi / 2)$}

For the hemispherical bubbles seen in regime I (see top photographs in Fig. 2 and Movie S1 in the Supplementary Material ${ }^{22}$ ), Bernoulli's relation written along the vertical streamline between the apex of the bubble and the center of the orifice reads $P_{0}+\frac{4 \gamma}{R_{c}}+\frac{\rho \dot{R}_{c}{ }^{2}}{2}=P_{0}+\frac{\rho v_{0}{ }^{2}}{2}$ where $P_{0}$ is the atmospheric pressure. ${ }^{6,25}$ It is worthwhile noticing that in contrast with Torricelli's problem ${ }^{1}$ which is driven by the hydrostatic pressure, the dynamics is driven in our case by the Laplace pressure $\frac{4 \gamma}{R_{c}}$. The Bernoulli's relation can be rearranged as $v_{0}^{2}={\dot{R_{c}}}^{2}+\frac{8 \gamma}{\rho R_{c}}$. As mentioned above, the conservation of the air flow rate gives $\dot{V}=2 R_{c}{ }^{2} \dot{R}_{c}=-a^{2} v_{0}$. Using this relation in the expression of the velocity $v_{0}$ determined using Bernoulli's principle, one readily finds the following first-order ordinary differential equation for
$R_{c}: \dot{R}_{c}=\frac{d R_{c}}{d t}=2 \sqrt{2} a^{2} \sqrt{\frac{\gamma}{\rho R_{c}}} \frac{1}{\sqrt{4 R_{c}^{4}-a^{4}}}$.

Using $\tau=\mathcal{G} \tau_{i n}$, where $\mathcal{G}=\left(\frac{R_{0}}{a}\right)^{2}$ is a geometric dimensionless quantity and $\tau_{i n}=\sqrt{\frac{\rho R_{0}{ }^{3}}{\gamma}}$ is an inertiocapillary timescale, this ODE can be re-written for $r_{c}$

$$
\dot{r_{c}}=\frac{d r_{c}}{d \varepsilon}=2 \sqrt{\frac{2}{r_{c}}} \frac{\mathcal{G}}{\sqrt{4 \mathcal{G}^{2} r_{c}^{4}-1}}
$$

which can be solved numerically. Interestingly, the ODE can take an even simpler form that can be solved analytically when we make an assumption easy to meet experimentally. Within the limit $\mathcal{G}=\left(\frac{R_{0}}{a}\right)^{2}=-\frac{v_{0}}{2 \dot{R}_{c}} \gg$ 1 , it is indeed reasonable to consider that the term $\dot{R}_{c}{ }^{2}$ is negligible compared to $v_{0}^{2}$ in the Bernoulli relation so that the ODE takes the form

$$
\dot{r}_{c} r_{c}{ }^{5 / 2}=-\sqrt{2}
$$

which has an analytical solution, that is, $r_{c}{ }^{7 / 2}$ should decrease linearly with the normalized time $\varepsilon=t / \tau$ as

$$
r_{c}{ }^{7 / 2}=1-\frac{7}{\sqrt{2}} \varepsilon
$$

In other words, when $\mathcal{G}=\left(\frac{R_{0}}{a}\right)^{2} \gg 1$, the time $t_{\text {end }}$ at which all air has escaped out of a hemispherical bubble should be $t_{\text {end }}=\frac{\sqrt{2}}{7} \tau \simeq 0.202\left(\frac{R_{0}}{a}\right)^{2} \sqrt{\frac{\rho R_{0}}{\gamma}}$. These predictions are compared to experiments in section IV.

\section{B. Regime II: the case of spherical caps $(\theta<\pi / 2)$ with a moving bubble's foot}

When $\theta<\pi / 2$, as shrinking proceeds (see middle sequence images in Fig. 2 and Movie S2 in the Supplementary Material $\left.{ }^{22}\right)$, the bubble's shape is a spherical cap having a volume $\frac{\pi}{3} H^{2}\left(3 R_{c}-H\right)$. The conservation of the air flow rate now gives the relation $v_{0}=$ $-\frac{H}{a^{2}}\left[\dot{H}\left(2 R_{c}-H\right)+H \dot{R}_{c}\right]$. Bernoulli's principle written along the vertical streamline between the bubble's apex and the center of the orifice gives $v_{0}^{2}=\frac{8 \gamma}{\rho R_{c}}+\dot{H}^{2}$. Combining the two expressions of the air velocity $v_{0}$, non-dimensionalizing as mentioned in the previous section and using $\tau=\mathcal{G} \tau_{i n}$ as in the case of hemispherical bubbles, one easily obtains

$$
h\left[\dot{h}\left(2 r_{c}-h\right)+h \dot{r}_{c}\right]=-\frac{1}{\mathcal{G}} \sqrt{\dot{h}^{2}+\frac{8 \mathcal{G}^{2}}{r_{c}}} .
$$

To close the problem, one needs to write a second equation relating $\dot{r}_{c}, r_{c}, \dot{h}$ and $h$. Such an equation is provided by taking into account viscous dissipation that occurs at 


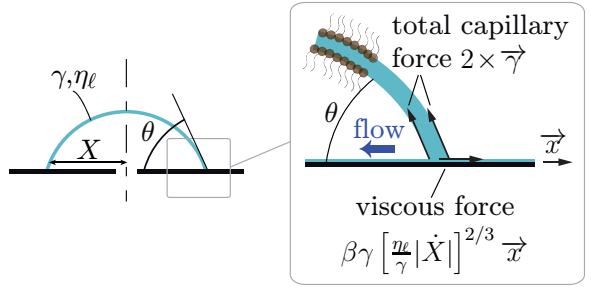

FIG. 4. Schematic of a surface soap bubble used in our study and close-up view of a bubble's foot illustrating the forces per unit foot length acting on it as discussed in the text. For the sake of clarity, this simplified view does not show the four surface tensions (two liquid-gas tensions and two liquidsolid ones) acting on the bubble's foot in the direction of the wetted solid. For each of the two pairs of phases (liquid-solid and liquid-gas), the two acting forces are indeed equal and opposite to each other so that they cancel one another out. The bubble sits on a thin film of liquid which is the same inside and outside the bubble's foot. Hence, it has no effect on the resultant forces. Also partially shown in the closeup view are the soap molecules covering the two liquid-air interfaces of the soap bubble.

the bubble foot moving on the wet solid. Viscous friction indeed explains the change in the dynamics seen between regime I and II (see Fig. 2): An initially hemispherical bubble becomes a spherical cap in regime II as its flattening dynamics is faster that the motion of its foot on the solid. Consequently, contrary to its variations in regime $\mathrm{I}, h$ is smaller than $r_{c}$ and $x$ at any time in regime II (see Fig. 3). Written per unit foot length, the viscous force acting on a bubble's foot in the direction of the solid plane varies nonlinearly with the foot's velocity and has the classical form $\beta \gamma\left[\frac{\eta_{\ell}}{\gamma}|\dot{X}|\right]^{2 / 3}$, where $\beta$ is a parameter that depends on boundary conditions. ${ }^{26,27} \mathrm{~A}$ capillary force with magnitude $2 \gamma$ is also acting on a bubble's foot in the direction of the bubble surface. Figure 4 shows a close-up view of a bubble's foot illustrating these forces. Considering quasistatic motion, the projection of the equilibrium forces onto $\vec{x}$ (Figure 4 defines the $\mathrm{x}$-axis) in the direction of the wet wall plane gives $2 \gamma \cos \theta=\beta \gamma\left[\frac{\eta_{\ell}}{\gamma}|\dot{X}|\right]^{2 / 3}$. Since $X$ is decreasing with time in regime II, we have $|\dot{X}|=-\dot{X}$. Also, because of geometry, $\cos \theta=\left(R_{c}-H\right) / R_{c}$ and $X^{2}+\left(R_{c}-H\right)^{2}=R_{c}{ }^{2}$. The derivation of the latter equation gives $\dot{X}=\left(\dot{R}_{c} H+\dot{H} R_{c}-\dot{H} H\right) / \sqrt{2 R_{c} H-H^{2}}$. Combining this expression and the balance of forces and non-dimensionazing with dimensionless quantities as in section III A, one finds the second equation

$$
h \dot{r_{c}}+\dot{h}\left(r_{c}-h\right)=-2 \sqrt{2} \mathcal{T}\left(1-\frac{h}{r_{c}}\right)^{3 / 2} \sqrt{h\left(2 r_{c}-h\right)}
$$

where $\mathcal{T}$ is the ratio of timescales $\mathcal{T}=\tau / \tau_{\text {vis }}$ with the visco-capillary timescale $\tau_{v i s}=\beta^{3 / 2} \eta_{\ell} R_{0} / \gamma$

The combination of Eq. (4) and Eq. (5) gives

$$
\left(h^{2} r_{c}{ }^{2}-\frac{1}{\mathcal{G}^{2}}\right) \dot{h}^{2}-2 I r_{c} h^{2} \dot{h}+\left(h^{2} I^{2}-\frac{8}{r_{c}}\right)=0
$$

where $I=2 \sqrt{2} \mathcal{T}\left(1-\frac{h}{r_{c}}\right)^{3 / 2} \sqrt{h\left(2 r_{c}-h\right)}$. This model is confronted to experiments in section IV.

\section{Regime III: the case of spherical caps $(\theta<\pi / 2)$ with a nonmoving bubble's foot}

As illustrated in the bottom images in Fig. 2 and in Movie S3 in the Supplementary Material, ${ }^{22}$ our experiments show that in regime III, seen for the smallest ratio $\left(\frac{R_{0}}{a}\right)^{2}$ and/or largest fluid dynamic viscosity $\eta_{\ell}$, the foot of a bubble barely moves throughout the shrinking process [see Fig. 3(a)]. Hence, we assume that it is pinned in this regime: $X=R_{0}$ and $\dot{X}=0$. In regimes II and III, a bubble's shape is a spherical cap whose volume written as a function of $H$ and $X$ using the geometric relation $R_{c}=\left(X^{2}+H^{2}\right) / 2 H$ is $\frac{\pi}{2} H\left(\frac{H^{2}}{3}+X^{2}\right)$. The conservation of the air flow rate gives $\dot{H}\left(H^{2}+X^{2}\right)=-2 a^{2} v_{0}$ where the velocity $v_{0}$ is obtained as in previous sections using Bernoulli's principle which reads $v_{0}^{2}=\frac{8 \gamma}{\rho R_{c}}+\dot{H}^{2}$. Contrary to the case of regime II in which the term $\dot{H}^{2}$ can take values that are not negligible when compared to the term $\frac{8 \gamma}{\rho R_{c}}$ given by the Laplace pressure, in regime III the former term is more that three orders of magnitude smaller than the latter; in Appendix B, Fig. B1 illustrates the comparison between the two terms for the data shown in Fig. 3(a). Consequently, one can approximately write $v_{0}=\sqrt{\frac{8 \gamma}{\rho R_{c}}}=4 \sqrt{\frac{\gamma H}{\rho\left(X^{2}+H^{2}\right)}}$ in regime III so that the flow rate conservation reads $\dot{H}=-8 a^{2} \sqrt{\frac{\gamma}{\rho}} \frac{\sqrt{H}}{\left(H^{2}+X^{2}\right)^{3 / 2}}$. Using the condition $X=R_{0}$ of regime III, non-dimensionalizing this relation, we find a prediction for the shrinkage time $t_{\text {end }}=\frac{1}{8} \mathcal{G} \tau_{\text {in }} \int_{0}^{1} \frac{\left(h^{2}+1\right)^{3 / 2}}{\sqrt{h}} d h$. Solving the integral numerically, we find $t_{\text {end }} \simeq 0.334 \mathcal{G} \tau_{\text {in }}$. We compare this prediction to experiments at the end of section IV.

\section{RESULTS AND DISCUSSION}

In this section, we compare the modeling work presented in section III to systematic experiments.

We begin by rationalizing the results found in regime I for which a bubble remains hemispherical as shrinking proceeds, i.e., $r_{c}=h=x$ (see the plot in the top panel in Fig. 3). Figure 5(a) shows the variations with time of the normalized radius of curvature $r_{c}$ in this regime for different soap solutions and radii of the orifice. For the presented set of experiments in Fig. 5, we work with small enough orifices and sufficiently large bubbles to fulfill the 

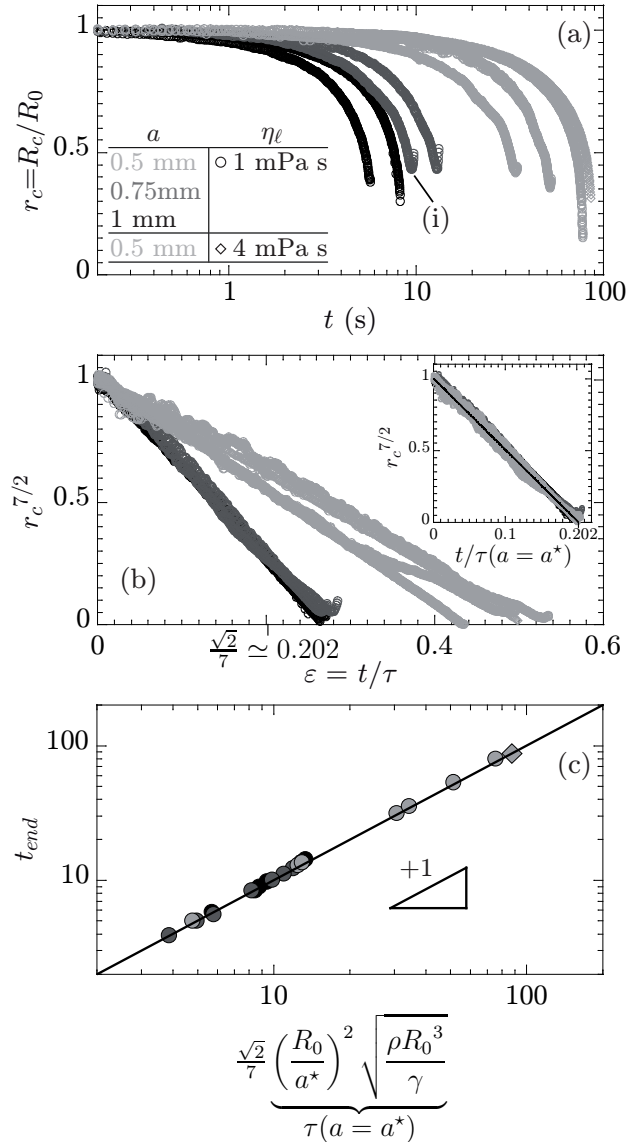

FIG. 5. (a) Variations with time of $r_{c}$ in regime I for different orifices and liquid viscosities as indicated. These experiments are performed with large enough bubbles and orifices not larger than a millimeter in radius so that $\mathcal{G}=\left(\frac{R_{0}}{a}\right)^{2} \gg 1$. As a result, the solution of Eq. (2) describes the variations of $r_{c}$ as, for each set of data, $r_{c}^{7 / 2}$ decreases linearly with the normalized time $\varepsilon$. Inset: All curves in the main plot collapse onto a single one when the effective radius $a^{\star}$ is used to determine $\tau$; this single curve is well-described by the black line which is a prediction calculated using Eq. (3) with $a=a^{\star}$. (c) As predicted, when $\mathcal{G} \gg 1$, the time at which the air has completely escaped out of the bubble is $t_{\text {end }}=\frac{\sqrt{2}}{7}\left(\frac{R_{0}}{a}\right)^{2} \sqrt{\frac{\rho R_{0} 3}{\gamma}}$. For these experiments, $\mathcal{G}$ varies in the $700-4200$ range.

condition $\mathcal{G}=\left(\frac{R_{0}}{a}\right)^{2} \gg 1$ which has allowed us to write the approximated ODE Eq. (2); in Fig. $5, \mathcal{G}=700-$ 4200. As a result, the temporal evolution of $r_{c}$ should be described by the solution of Eq. (2), that is, Eq. (3). As predicted by the latter equation, when $r_{c}^{7 / 2}$ is plotted as a function of the normalized time $\varepsilon$, this quantity is a linearly decreasing function of $\varepsilon$ [see Fig. 5(b)] for all data shown in Fig. 5(a). However, these curves do not collapse onto a single one as expected and the x-intercept of the predicted line $\left(\frac{\sqrt{2}}{7} \approx 0.202\right)$ is overestimated for each set of data [Fig. 5(b)]. Both predicted results are obtained only when an effective radius $a^{\star}$ is used instead of the real one $a$ to calculate $\tau$ [see inset of Fig. 5(b)]. Also, using $a=a^{\star}$, figure $5(\mathrm{c})$ shows that the experimental shrinkage time in regime I which varies over almost two orders of magnitude in our study is well-described by Eq. (3) which predicts $t_{\text {end }}=\frac{\sqrt{2}}{7} \tau \approx 0.202 \tau$.

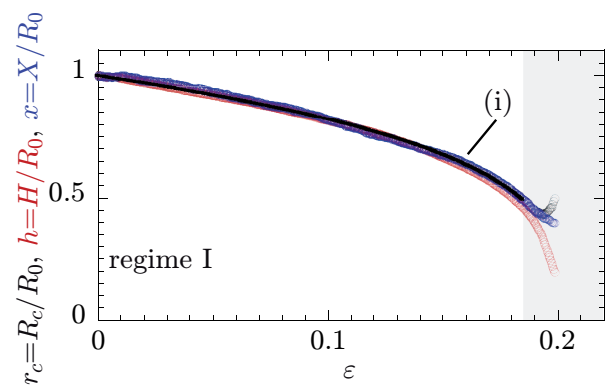

FIG. 6. Temporal evolutions of $r_{c}$, height $h$ and distance $x$ that correspond to the experiment denoted (i) in Fig. 4. As indicated by the gray area, the response is not that of regime I at late time: Friction effects come into play and the normalized distance are no longer identical. The line which corresponds to the prediction for regime I, $r_{c}=\left(1-\frac{7}{\sqrt{2}} \varepsilon\right)^{2 / 7}$, that is given by Eq. (3) with $a=a^{\star}$ rationalizes most of the dynamics.

As shown in Fig. $\mathrm{C} 1$ in Appendix $\mathrm{C}$, the effective radius $a^{\star}$ that is adjusted to compare experiments and modeling work appears to be slightly smaller than $a$. It is a weakly dependent function of the soap solution and it varies linearly with $a$ roughly as $a^{\star}=0.75 a$. It seems reasonable to find that an effective radius of the orifice smaller than the real one should be used to adjust the data since the speed of the air in the Bernoulli's relation used for each regime is written at the center of the orifice.

To summarize, for sets of hemispherical bubbles and orifices giving sufficiently large geometric dimensionless parameter $\mathcal{G}$, Figure $5(\mathrm{~b})$ and $(\mathrm{c})$ show that the shrinking dynamics are fully captured by the simple law given in Eq. (3) and that the prediction for $t_{\text {end }}$ well describes experimental findings when $a$ is a free parameter $a^{\star}$. The time $\tau$ will hereafter be estimated using $a^{\star}$.

Figure 6 shows the temporal evolution of the three normalized distances corresponding to the data denoted (i) in Fig. 5(a). For this set of data, the response is that of regime I for most of the dynamics, i.e., $r_{c}=h=x$ from $\varepsilon=0$ until the normalized time $\varepsilon$ is about 0.18 in Fig. 6. Friction at the bubble's foot comes into play at late times as illustrated by the gray domain displayed in 


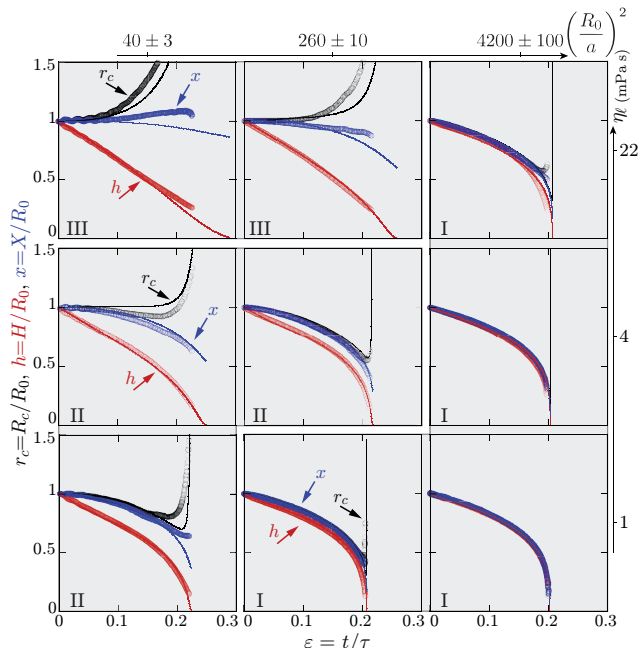

FIG. 7. Temporal variations of $r_{c}$, height $h$ and distance $x$ for different fluid dynamic viscosities $\eta_{\ell}$ and geometric ratios $\mathcal{G}=$ $\left(\frac{R_{0}}{a}\right)^{2}$ as indicated. Symbols stand for experiments and the lines are calculated using Eq. (6) and the method explained in the text.

Fig. 6. In this temporal domain, $r_{c} \neq h \neq x$ so that $\cos \theta=1-h / r_{c}>0$. As discussed in section III B to rationalize these shrinking dynamics, we use MATLAB to solve numerically Eq. (6) for $\dot{h}$. Equation (5) is then used to determine $\dot{r}_{c}$. Our software written with MATLAB finds values for $h$ and $r_{c}$ using Euler's method and $x$ is then determined using the geometric relation between the three distances, i.e., $x=\sqrt{h\left(2 r_{c}-h\right)}$. Figure 7 shows the temporal variations of the distance $r_{c}, h$ and $x$ with $\varepsilon$ for three different geometric ratios $\left(\frac{R_{0}}{a}\right)^{2}$ and the three fluids used in this study as indicated in the figure. The combination of these nine plots summarizes our study as they demonstrate the transitions between the three regimes seen experimentally are controlled by both the geometric ratio and the fluid dynamic viscosity. In addition, in Fig. 7, the lines calculated with our model concur well with the experimental data represented by symbols for the three regimes. To obtain a good agreement between experiments and predictions, $\beta$ is introduced as a second free parameter of the model discussed in section IIIB. We find that the value taken by $\beta=40 \pm 8$ is independent of the liquid used and the investigated regime. $\beta$ is however larger than the value usually reported in the literature: Depending on whether the air-liquid interface is mobile or rigid, $\beta \simeq 5-6 .{ }^{26,27}$ The larger value of $\beta$ taken in our experiments could be explained be the strongly rigid interfaces often found in commercial detergent solutions which would enhanced the viscous dissipation at the moving bubble foot. ${ }^{28}$

Equation (5) reveals that in fact the governing di-

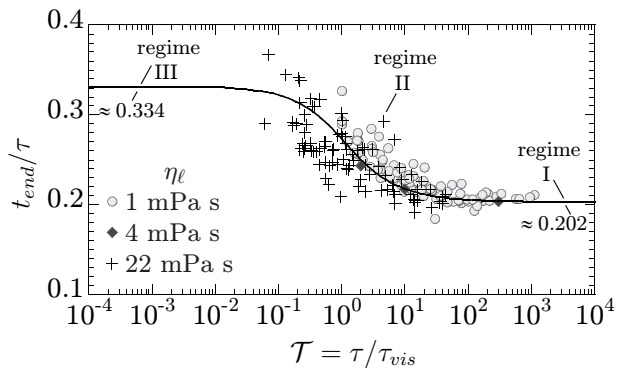

FIG. 8. Variations of the ratio $t_{\text {end }} / \tau$ between experimental shrinkage time and predicted characteristic time with the controlling dimensionless time $\mathcal{T}=\tau / \tau_{\text {vis }}$. The symbols stand for experimental data and the solid line is calculated using our model.

mensionless parameter of the problem is $\mathcal{T}=\tau / \tau_{\text {vis }}=$ $\mathcal{G} \tau_{\text {in }} / \tau_{\text {vis }}$ which does not only comprise the discussed geometric ratio $\mathcal{G}=\left(\frac{R_{0}}{a}\right)^{2}$ and dynamic viscosity $\eta_{\ell}$ used in Fig. 7 but also other parameters of the problem via the inertio-capillary $\left(\tau_{i n}=\sqrt{\frac{\rho R_{0}^{3}}{\gamma}}\right)$ and visco-capillary $\left(\tau_{v i s}=\beta^{3 / 2} \eta_{\ell} R_{0} / \gamma\right)$ timescales. As shown in Fig. $8, \mathcal{T}$ is the parameter that should be tuned to modify the response from regime I, to regime II, to regime III. Indeed, this figure showing the variations of $t_{\text {end }} / \tau$ with $\mathcal{T}$ summarizes well our findings as the roughly 200 experiments that were performed and analyzed in the three shrinking regimes collapse onto a single curve that is welldescribed by the solid line calculated using our model. Additionally, although it is difficult to perform experiments when $\mathcal{T}$ is smaller than about $10^{-1}$, we find that the predicted expression $t_{\text {end }} / \tau \simeq 0.334$ for regime III (see section IIIC) emerges from numerical simulations and correlates with experimental data in this regime (i.e., data points in the range $\mathcal{T} \simeq 0.1-0.3$ in Fig. 8); we could not perform experiments with values of $\mathcal{T}$ smaller than about $10^{-1}$ because it is difficult to prepare bubbles with smaller ratios $R_{0} / a$ and/or larger liquid viscosities $\eta_{\ell}$ than those reported.

\section{CONCLUSIONS}

We have studied the hitherto uninvestigated shrinkage of centimeter-sized surface bubbles. Our results have revealed three distinct shrinking scenarios, the occurrence of which is controlled by a dimensionless parameter that is a function of the physicochemistry of the fluid system and the geometric variables at play. In the first two scenarios, a bubble's foot recedes when shrinking occurs, the bubble either remaining hemispherical or seeking the shape of a spherical cap during the process. In the third shrinking scenario that we have identified, a bubble flattens with a nonmoving bubble's foot as shrinking pro- 
ceeds. Contrary to the classical problem of the draining of a tank through an orifice controlled by the hydrostatic pressure, the driving force is given by the Laplace pressure in the case of shrinking bubbles and we show that the process is limited by viscous dissipation acting at a bubble's foot. These physical arguments combined with conservation of air flow rate allow us to present a model that rationalizes the shrinking dynamics seen in the three scenarios. As discussed in the article, this experiment would make a great project for undergraduate and graduate physics courses. First of all, experiments can be performed by students without the need for sophisticated equipment. In addition, the first regime is described by an ODE that has an analytical solution when making a reasonable approximation. As the resulting predictions for the temporal variations of a bubble size and time at which shrinking ends concur well with experiments, a project based on the study of the first regime should also teach students the usefulness of well-justified approximations in physics. Also, upper-division students could benefit from the experiment and particularly from the case of shrinking spherical caps. This case is indeed described by an ODE that can be solved numerically making it a good combination of experimental and computational work.

\section{ACKNOWLEDGMENTS}

We thank Franck Artzner for the rheometry that we used to determine the fluid viscosities and Benjamin Reichert and Arnaud Saint-Jalmes for the surface tension measurements. We also thank Isabelle Cantat and Benjamin Dollet for fruitful discussions.

\section{Appendix A: Description of the movies ${ }^{22}$}

- Movie S1 - Shrinking quasi-hemispherical bubbles in regime I: A bubble remains quasi-hemispherical as shrinking proceeds in regime I. These observations are made with a low-viscosity fluid soap solution $\left(\eta_{\ell}=\right.$ $1 \mathrm{mPa} \mathrm{s})$, a sufficiently large initial bubble $\left(R_{0}=\right.$ $32.68 \mathrm{~mm})$ and a small orifice $(a=0.5 \mathrm{~mm})$.

- Movie S2 - The case of shrinking spherical caps in regime II: In regime II, a bubble seeks the shape of a spherical cap as air escapes through the orifice. The viscous effects acting on a bubble's foot are important in this regime illustrated for $\eta_{\ell}=1 \mathrm{mPa} \mathrm{s}$, a bubble having an initial radius $R_{0}=31.66 \mathrm{~mm}$ and a value of $a=2 \mathrm{~mm}$ that is four times larger than that of Movie S1.

- Movie S3 - Collapsing bubbles with a nonmoving bubble foot in regime III: For large enough liquid viscosities $\left(\eta_{\ell}=22 \mathrm{mPa} \mathrm{s}\right.$ in the experiment shown in Movie S3) and sufficiently small ratio between bubble and orifices radii $\left(R_{0}=33.1 \mathrm{~mm}\right.$ and $\left.a=5 \mathrm{~mm}\right)$, Movie $\mathrm{S} 3$ shows that the dynamics is characterized by a bubble collapsing on itself with a nonmoving bubble's foot.
Appendix B: Comparison between the terms $\dot{H}^{2}$ and $\frac{8 \gamma}{\rho R_{c}}$ of the Bernoulli's relation in regime III

Figure B1 shows that $\dot{H}^{2}$ is more than three order of magnitude smaller than the term $\frac{8 \gamma}{R_{2}}$ given by the Laplace pressure in regime III. These data correspond to the results shown in Fig. 3(a) of the text.

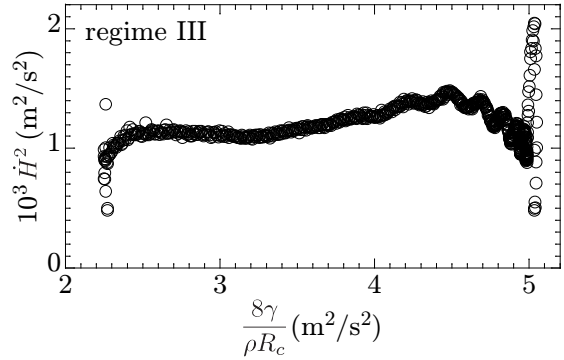

FIG. B1. Evolution of $\dot{H}^{2}$ with $\frac{8 \gamma}{\rho R_{c}}$ in regime III for the data shown in Fig. 3(a).

Appendix C: Relation between measured radius $a$ and its effective value $a^{\star}$

As shown in Fig. C1 the values taken by the free parameter $a^{\star}$ collapse onto a single curve when plotted as a function of $a$ : $a^{\star}$ which is weakly dependent on the fluid system varies linearly with $a$ roughly as $0.75 a$.

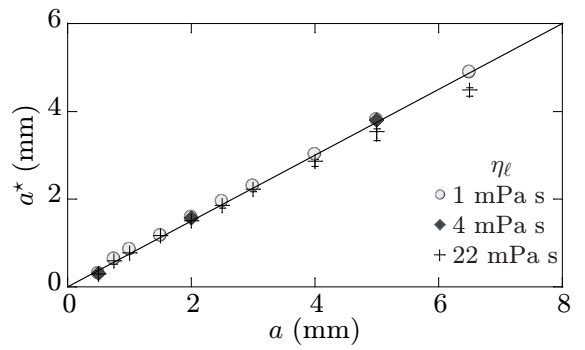

FIG. C1. Variations of the effective radius of the orifice $a^{\star}$ as a function of its measured value $a$ for the three soap solutions as indicated. The line is a guide for the eyes that corresponds to the equation $a^{\star}=0.75 a$. 
* These two authors contributed equally to the work

$\dagger$ laurent.courbin@univ-rennes1.fr

¥ pascal.panizza@univ-rennes1.fr

${ }^{1}$ E. Torricelli, Opera Geometrica, De Sphaera et Solidis Sphaeralibus; De Motu Gravium: De Dimensione Parabolae (Amadoro Massa \& Lorenzo de Landis, Florence, 1644).

2 J. Ferrand, L. Favreau, S. Joubaud, and E. Freyssingeas, "Wetting effect on Torricelli's law," Phys. Rev. Lett. 117, 248002-1-5 (2016)

3 D.P. Jackson and S. Sleyman, "Analysis of a deflating soap bubble," Am. J. Phys. 78, 990-994 (2010).

${ }^{4}$ J. Plateau, Statique expérimentale et théorique des liquides soumis aux seules forces moléculaires (Gauthier-Villars, Paris, 1873).

${ }^{5}$ H. Bouasse, Capillarité-Phénomènes superficiels (Librairie Delagrave, Paris, 1924).

${ }^{6}$ P. G. de Gennes, F. Brochard-Wyart, and D. Quéré, Cap illarity and Wetting Phenomena: Drops, Bubbles, Pearls, Waves (Springer, New-York, 2004).

7 L. Salkin, A. Schmit, P. Panizza, and L. Courbin, "Generating soap bubbles by blowing on soap films," Phys. Rev. Lett. 116, 077801-1-5 (2016).

${ }^{8}$ P. Panizza and L. Courbin, "Bubble blowing by the numbers," Phys. Today 69(7), 78-79 (2016).

9 B. Dollet, P. Marmottant, and V. Garbin, "Bubble dynamics in soft and biological matter," Annu. Rev. Fluid Mech. 55, 331-355 (2019).

$10 \mathrm{~J} . \mathrm{Wu}$, "Evidence of sea spray produced by bursting bubbles," Science 212, 324-326 (1981).

11 A. H. Woodcock, C. F. Kientzler, A. B. Arons, and D. C. Blanchard, "Giant condensation nuclei from bursting bubbles," Nature 172, 1144-1145 (1953).

12 F. MacIntyre, "Flow patterns in breaking bubbles," Nature $\mathbf{7 7}, 5211-5228$ (1972).

13 J. C. Bird, R de Ruiter, L. Courbin, and H. A. Stone, "Daughter bubble cascades produced by folding of ruptured thin films," Nature 465, 759-762 (2010).

${ }^{14} \mathrm{H}$. Lhuissier and E. Villermaux, "Bursting bubble aerosols," J. Fluid Mech. 696, 5-44 (2012).

15 L. Champougny, M. Roché, W. Drenckhan, and E. Rio, "Life and death of not so "bare" bubbles," Soft Matter 12, 5276-5284 (2016).

16 S. Poulain and L. Bourouiba, "Disease transmission via drops and bubbles," Phys. Today 72(5), 70-71 (2019).

17 F. Seychelles, Y. Amarouchene, M. Bessafi, and H. Kellay, "Thermal Convection and Emergence of Isolated Vortices in Soap Bubbles", Phys. Rev. Lett. 100, 144501-1-4 (2008).

18 T. Meuel, Y. L. Xiong, P. Fischer, C. H. Bruneau, M. Bessafi and H. Kellay, "Intensity of vortices: from soap bubbles to hurricanes", Sci. Rep. 3, 3455-1-7 (2013).

19 L. Salkin, A. Schmit, R. David, A. Delvert, E. Gicquel, P. Panizza, and L. Courbin, "Interfacial bubbles formed by plunging thin liquid films in a pool," Phys. Rev. Fluids 2 063604-1-10 (2017)

$20<$ https://physlets.org/tracker/>

$21<$ https://imagej.nih.gov/ij/>

${ }^{22}$ An annotated script of the image processing software written with MATLAB and three movies (Movie S1, S2 and S3) that illustrate the three regimes seen experimentally can be found here, i.e., <https://drive.google.com/drive/folders/ 1W5m3jq9D-gB9Cnm3HU18GViCTtuuFT31?usp=sharing>.

We provide a description of these movies in Appendix A.

${ }^{23}$ S. Arscott, "Wetting of soap bubbles on hydrophilic, hydrophobic, and superhydrophobic surfaces," Appl. Phys. Lett. 102, 254103-1-4 (2013).

${ }^{24}$ L. Salkin, A. Schmit, P. Panizza, and L. Courbin, "Influence of boundary conditions on the existence and stability of minimal surfaces of revolution made of soap films," Am. J. Phys. 82(9), 839-847 (2014).

25 In the case of a spherical drop of radius $R$, the well-known expression of the Laplace pressure is $2 \gamma / R$. For bubbles, the factor 2 in the overpressure $2 \times 2 \gamma / R_{c}$ that appears in the Bernoulli's relation is due to the presence of two liquid-gas interfaces as illustrated in Fig. 4.

${ }^{26}$ B. Dollet and I. Cantat, "Deformation of soap films pushed through tubes at high velocity," J. Fluid Mech. 652, 529539 (2010).

27 I. Cantat, "Liquid meniscus friction on a wet plate: Bubbles, lamellae and foams." Phys. Fluids 25, 031303-1-21 (2013).

${ }^{28}$ I. Cantat, private communication. 